\title{
Design of a Fuzzy Inference Based Ultrasound Image Analysis System for Differential Diagnosis of Thyroid Nodules
}

\author{
Poornima $\mathrm{D}^{1, *}$ Asha Gowda Karegowda ${ }^{2}$, Pushpalatha K.R ${ }^{3}$
}

\author{
${ }^{1}$ RRIT, Bangalore, India \\ ${ }^{2}$ SIT, Tumakuru, India \\ ${ }^{3}$ SSIT, Tumakuru, India \\ *Corresponding author. Email: poornimarajud@gmail.com
}

\begin{abstract}
This paper presents a Fuzzy Inference based Ultrasound Image Analysis System for differential diagnosis of Thyroid Nodules (TNs). Thyroid Ultrasound (TUS) images containing TNs are preprocessed to remove speckle noise and are enhanced using histogram equalization method. Nodule boundaries are identified using the canny edge detection technique and required Region of Interest is obtained using Adaptive Regularized Kernel Fuzzy C-means (ARKFCM) segmentation algorithm. Nineteen texture features are extracted from the segmented images. Best First (BF), Genetic Search (GS) and Greedy Step Wise (GSW) search methods are applied to select best subset of features. Selected features are fuzzified. A novel, fuzzy system is built to discriminate benign from malignant TNs by employing Mamdani model to draw inferences and centroid scheme for defuzzification. Class Based Association (CBA) concept is used to generate fuzzy inference rules. The developed multiple input, single output FIUIAS resulted in an accuracy of $98 \%$.
\end{abstract}

Keywords: Classification, Feature Selection, Fuzzy inferencing, Medical Image processing, Thyroid nodule.

\section{INTRODUCTION}

Medicine is one of the fastest growing field, with several degrees of uncertainty and vague nature of decision making. Medical practice is often complex due to imprecise and unavailability of information. Rise of modern medicine, rapid developments in diagnostic procedures and treatments, and advances in digital information processing have contributed more to complexity of medical practice [1]. Since imperfect knowledge is inevitable in medicine, modelling medical information systems requires employing soft computing techniques such as evolutional computation, fuzzy inference systems, probabilistic reasoning, neural networks and swam intelligence techniques [2]. Complex medical data can be analyzed through the application of different creative technologies and strategies such as fuzzy logic or in combination with other soft computing techniques. Fuzzy frameworks are applied to each area of medicine and they are proven to be successful [3]. Few applications of fuzzy methods applied to medical field include knowledge acquisition, dealing with inconsistencies, treatment planning and artificial thinking [4].

Rapid advances in different diagnostic imaging techniques enable us to acquire high-dimensional, sectional, thin-sliced, and a large number of images within a short acquisition time. The sheer volume of data poses great challenges for human interpretation of images by radiologists [5]. Every day, imaging techniques are developing. Image processing becomes essential to analyse such complex data. Current developments in the computerized analysis of medical images are expected to assist radiologists and other health care professionals in various diagnostic decisions of medical image interpretation [6].

In the present work, a fuzzy Inference based ultrasound Image analysis system (FIUIAS) is designed to aid in the differential diagnosis of TNs. 
Solid or fluid filled TNs are the lumps formed within the thyroid gland. Vast majority of TNs are benign, non-cancerous and do not cause symptoms. Benign nodules are very common in elderly population or people with iodine deficiency [7]. Small percentage (10\%) of TNs are malignant meaning cancerous. The present study focused on applying fuzzy concepts to classify TNs.

The rest of the paper is organized as follows. Section 2 discusses the related research work carried out to classify TNs using different machine learning, deep learning and image processing techniques, Section 3 explains the proposed methodology adopted to build FIUIAS followed by experimental results and conclusion in Section 4 and 5 respectively.

\section{RELATED WORK ON THYROID NODULE CLASSIFICATION}

Many authors have worked on TN segmentation and classification using different machine learning and image processing techniques.

Maroulis et al. [8] developed an original Variable Background Active Contour (VBAC) technique to segment TNs in TUS images. The performance of VBAC is compared with Active Contour without Edges (ACWE) model in terms of mean overlap value and average segmentation time and results are verified with manual delineation by expert radiologists. From the results of experiments, it was concluded that VBAC gave accurate delineations of hyperechoic nodules compared to ACWE and in fewer iterations.

Iakovidis et al. [9] presented a segmentation technique, GA based VBAC for precise mark of TNs from TUS images. The results of GA-VBAC are compared with the results obtained by expert radiologists in terms of average overlap value. It was demonstrated from the experiments that the segmentation result of GA-VBAC is equivalent to or better than the segmentation result obtained by concerned radiologist and inter observer variability of radiologist is greater than the variability of the overlap values obtained with GA-VBAC.

Selvathi et al. [10] designed a CADD system using wavelet transform and SVM with polynomial kernel to delineate TG using TUS images. Horizontal projection is used to identify the thyroid region and Adaptive Weighted Median Filter (AWMF) is applied to reduce SN. Statistical features mean and variance are extracted from the ROI using Haar wavelet transform. These features are used to develop the SVM to separate thyroid region from the rest of area. The corresponding segmentation outputs of SVM are tested with segmentation results of expert. Accuracy for results using statistical features extracted from wavelet transform is higher than accuracy for results obtained without using wavelet transform identified features.

Bibicu et al. [11] proposed an innovative method to automatically detect TNs from thyroid echography pictures. The algorithm is extended to images containing ROIs like TN, thyroid region and wind pipe. The efficacy of the innovative algorithm is carried using sensitivity, specificity, correct classification rate, and ROC analysis have been considered. For complete thyroid echography pictures, the obtained accuracy to recognize TNs is $82.99 \%$. But for ROI extracted images the accuracy is $91.01 \%$ clearly demonstrating the use of segmentation for considered images.

Keramidas et al. [12] planned a new technique to automatically identify TNs using features derived from longitudinal TUS images. Hyperechoic lines defining ROI are identified using a new algorithm, Thyroid Boundaries Detection (TBD). From this ROI, relevant features are extracted and same features are used to carry out classification. Outcome of TBD results are compared with exhaustive feature extraction technique. Authors concluded that proposed technique improved nodule detection accuracy while decreasing the processing time considerably.

Chi et al. [13] presented a complete computerized diagnosis system using deep learning approach to classify TNs in TUS images. These images are preprocessed to normalize the texture scales and remove artifacts. Also, new approach to augmentation is executed by cropping thyroid samples from image regions around the center of certain TN. A pre-trained Google Net model is fine tuned to extract better features. These features are input to supervised Random Forest classifier. The performance was evaluated using classification accuracy, sensitivity, specificity and area under receiver operating characteristic curve. The experimental results show the proposed fine-tuned GoogLeNet model achieved excellent classification performance.

Keramidas et al. [14] used Fuzzy Logic (FL) and Local Binary Patterns (LBP) to implement a new technique, FL-LBP to analyze and evaluate a texture representation in TUS images. FL-LBP improved texture representation and is unaffected by $\mathrm{SN}$ which is natural in TUS images. FL-LBP results is compared with radon transformation, co-occurrence matrix and crisp LBP. FL-LBP results are also cross verified using SVM with polynomial kernel. The classification results for all experiments were computed by 10 -fold CV method. Better results were achieved using FL-LBP when compared to 
other considered techniques. It was deduced that the proposed technique can be employed by medical staff for TUS image scanning and diagnosis.

\section{DESIGN OF FUZZY INFERENCE BASED ULTRASOUND IMAGE ANALYSIS SYSTEM (FIUIAS)}

The design of proposed FIUIAS is depicted in Figure 1. Both online and clinical nodular Thyroid Ultrasound Images (TUS) are used to carry out the experiment. The system is implemented using Matlab R2014a software [15].

\subsection{Image Collection}

Thyroid ultrasound is the optimal initial imaging method in the screening of TNs due to its high resolution, low cost, availability, non-invasivity and no radiation load [16]. Ultrasound is highly sensitive for detection of thyroid nodules and features like size, shape, location, composition, echogenicity, margins and vascularity of nodules obtained can be used for further investigation [17]. Ultrasound images containing benign and malignant thyroid nodules are used in the present study to design and test fuzzy system. TUS images were procured from NRR hospital, Bangalore. A total of 200 images (150 benign, 50 malignant) are considered in this study. Along with these images an open access database of thyroid US images containing benign and malignant nodules are also used [18]. A total of 150 images (100 benign, 50 malignant) are considered in this study.

\subsection{TUS image pre-processing}

Ultrasound images are generally affected by multiplicative speckle noise [19]. In this work, TUS images are preprocessed in two stages. In the first stage, speckle filtering is carried out using four filters namely: lee, frost, kuan and median filter. The filtered images using Kuan filter are further used to carry out experiment since it resulted in higher PSiNR and lower MeSE compared to other applied filters (Table 1). In the second stage the contrast between the thyroid nodule and background is enhanced using histogram equalization method and nodule boundaries are identified using the canny edge detection technique.

\subsection{TUS image segmentation}

Accurate delineation of benign and malignant thyroid nodules is carried out using FCM, K-means, PSO and ARKFCM based segmentation techniques. The efficiency of applied segmentation algorithms is measured in terms of (average values of) Peak Signal to Noise Ratio (PSiNR), Mean Square Error (MeSE), Normalized Cross Correlation (NCrC), Structural Similarity Index (StSI), segmentation accuracy and time taken for segmentation [20]. ARKFCM accurately delineated the nodules compared to PSO, FCM and K-means and hence images segmented using ARKFCM are further used to carry out the experiment. The denoised image using Kuan filter (best filter), canny edge detection image and segmented image using ARKFCM is portrayed in Figure 2.

\subsection{Feature Extraction}

From each segmented image 19 textural features were extracted. Extracted features include autocorrelation, clusterProminence, clusterShade, contrast, correlation, differenceEntropy, differenceVariance, dissimilarity, energy, entropy, homogeneity, informationMeasureOfCorrelation1, informationMasureOfCorrelaton2,

inverseDifference, maximumProbability, sumAverage, sumEntropy, sumOfSquaresVariance, and sumVariance [21].

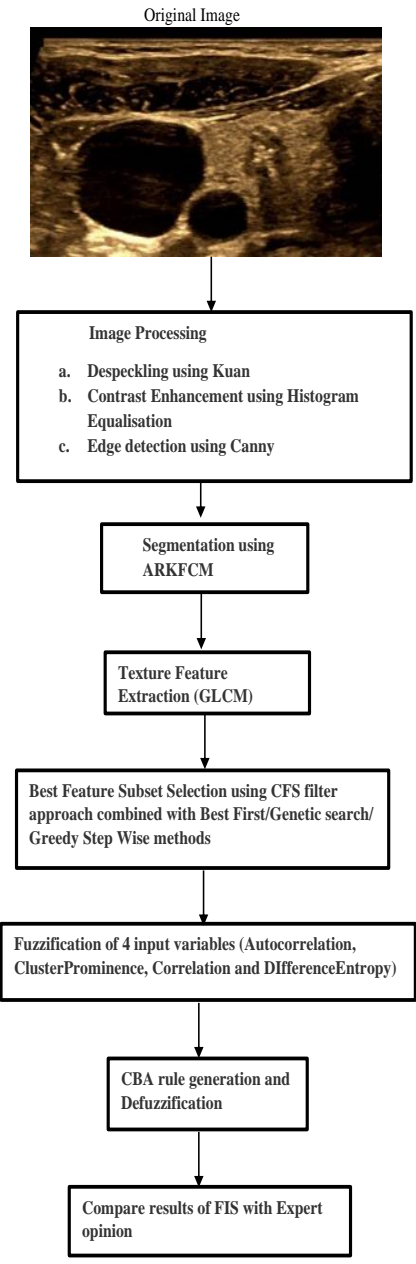

Figure 1 Proposed FIUIAS design 
Table 1 Performance comparison of different filters

\begin{tabular}{|l|l|}
\hline \multicolumn{1}{|c|}{ Filter } \\
\hline $\begin{array}{l}\text { Speckle noisy } \\
\text { image }\end{array}$ \\
\hline Lee & \\
\hline & \\
\hline & \\
\hline &
\end{tabular}

(a)

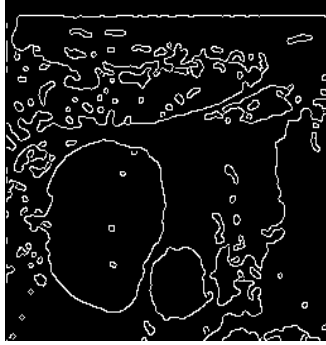

(b)
Figure 2 (a) denoised image

\begin{tabular}{|l|l|l|}
\hline Frost & 26.97 & 130.68 \\
\hline Kuan & $\mathbf{4 4 . 9 7}$ & $\mathbf{2 . 0 7}$ \\
\hline Median & 29.42 & 74.37 \\
\hline
\end{tabular}

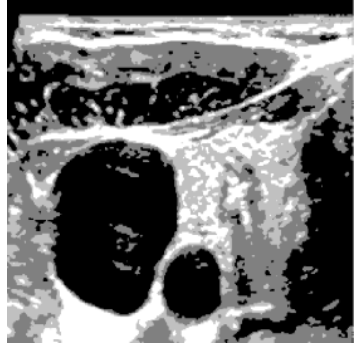

(c)

(c) Segmented image

\subsection{Feature Selection}

Efficient feature selection technique is needed to extract interesting features from medical datasets as these data sets contain huge number of redundant and irrelevant features [22]. The testing and training of classification systems becomes difficult for datasets of higher dimension. In this paper, three search methods namely BF, GS and GSW are used to generate candidate feature subset using 19 features and selected features are evaluated using Correlation based Feature Subset (CFS) selection filter approach. Features selected using CFS filter approach for 3 different search methods include autocorrelation, clusterProminence, correlation, and differenceEntropy. Feature values are stored in excel sheet and a sample for ten TUS images is shown in Table 2. Proposed FIUIAS is implemented using these four features as input and output of FIUIAS is a class label indicating whether the nodule is benign or malignant [36-39].

Table 2. Feature values extracted from TUS images

\begin{tabular}{|l|l|l|c|}
\hline $\begin{array}{l}\text { Autocorr } \\
\text { elation }\end{array}$ & $\begin{array}{l}\text { ClusterPro } \\
\text { minence }\end{array}$ & $\begin{array}{l}\text { Correl } \\
\text { ation }\end{array}$ & $\begin{array}{l}\text { Difference } \\
\text { Entropy }\end{array}$ \\
\hline 230.64 & 11.45199 & 0.099 & 0.194 \\
\hline 366.09 & 19.59828 & 0.085 & 0.152 \\
\hline 228.46 & 13.64 & 0.130 & 0.238 \\
\hline 273.06 & 2.58 & 0.112 & 0.227 \\
\hline 321.54 & 11.53 & 0.105 & 0.177 \\
\hline 296.67 & 17.39 & 0.127 & 0.228 \\
\hline 323.88 & 9.30 & 0.117 & 0.189 \\
\hline 329.13 & 17.92 & 0.104 & 0.191 \\
\hline 327.62 & 20.42 & 0.137 & 0.245 \\
\hline 348.54 & 29.34 & 0.130 & 0.247 \\
\hline
\end{tabular}

\subsection{Fuzzification}

The corresponding crisp values of four features selected in the previous step is transformed to linguistic terms using triangular membership functions [23]. Table 3 shows converted equivalent linguistic terms for the corresponding discrete data in Table 2.

Table 3. Linguistic variables corresponding to input variables

\begin{tabular}{|c|c|c|c|}
\hline $\begin{array}{l}\text { Autocorr } \\
\text { elation }\end{array}$ & $\begin{array}{l}\text { ClusterPro } \\
\text { minence }\end{array}$ & $\begin{array}{l}\text { Correl } \\
\text { ation }\end{array}$ & $\begin{array}{l}\text { Difference } \\
\text { Entropy }\end{array}$ \\
\hline $\mathrm{lw}$ & $\mathrm{lw}$ & $\mathrm{lw}$ & $\mathrm{lw}$ \\
\hline $\mathrm{lw}$ & $\mathrm{lw}$ & $\mathrm{md}$ & $\mathrm{md}$ \\
\hline $\mathrm{lw}$ & $\mathrm{lw}$ & $\mathrm{md}$ & $\mathrm{md}$ \\
\hline $\mathrm{lw}$ & $\mathrm{md}$ & $\mathrm{md}$ & $\mathrm{md}$ \\
\hline $\mathrm{lw}$ & $\mathrm{md}$ & $\mathrm{md}$ & $\mathrm{md}$ \\
\hline $\mathrm{md}$ & $\mathrm{lw}$ & $\mathrm{lw}$ & $\mathrm{md}$ \\
\hline $\mathrm{md}$ & $\mathrm{lw}$ & $\mathrm{lw}$ & $\mathrm{lw}$ \\
\hline $\mathrm{lw}$ & $\mathrm{lw}$ & $\mathrm{lw}$ & $\mathrm{lw}$ \\
\hline $\mathrm{hi}$ & $\mathrm{hi}$ & $\mathrm{hi}$ & $\mathrm{hi}$ \\
\hline \multicolumn{5}{|c|}{$\mathrm{hi}$} & $\mathrm{hi}$ & $\mathrm{md}$ & $\mathrm{md}$ \\
\hline \multicolumn{5}{|c|}{$*$ lw- low, md-mid, hi- high } \\
\hline
\end{tabular}

The graphical representation of fuzzy membership functions for autocorrelation, clusterProminence, correlation, differenceEntropy and class benign/malignant nodule is shown in figures $3,4,5,6$ and 7 respectively.

\subsection{Fuzzy rule generation}

In this study, WEKA tool is employed to produce CBA rules [24]. Association rule mining 
finds all the rules existing in the database that satisfy some minimum support and minimum confidence whereas CBA aims to discover a small set of rules in the database that forms an accurate classifier. A collection of standard 40 rules with $3 \%$ support and $100 \%$ confidence were created and sample rule set is shown in Figure $8 \& 9$ for benign and malignant class respectively. These rules were examined to verify that they cover all possible combinations of inputs and outputs. [33-35]

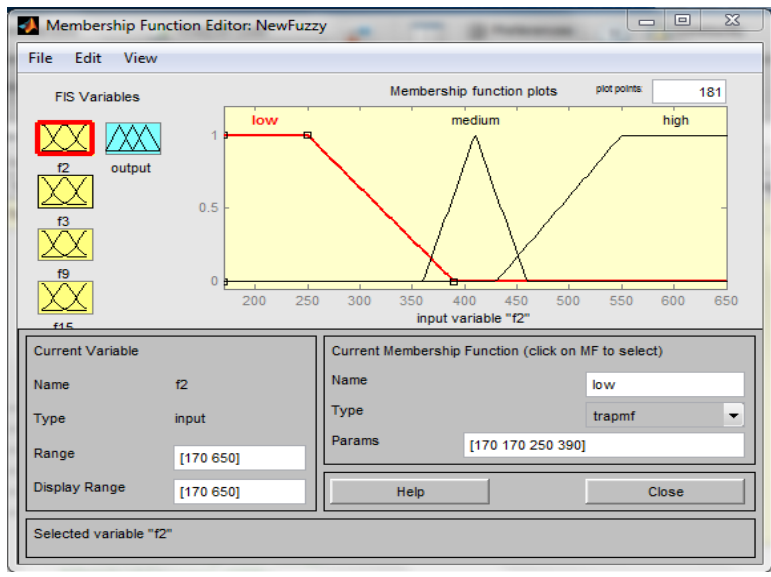

Figure 3 Membership function for feature f2

\section{i. e Autocorrelation}

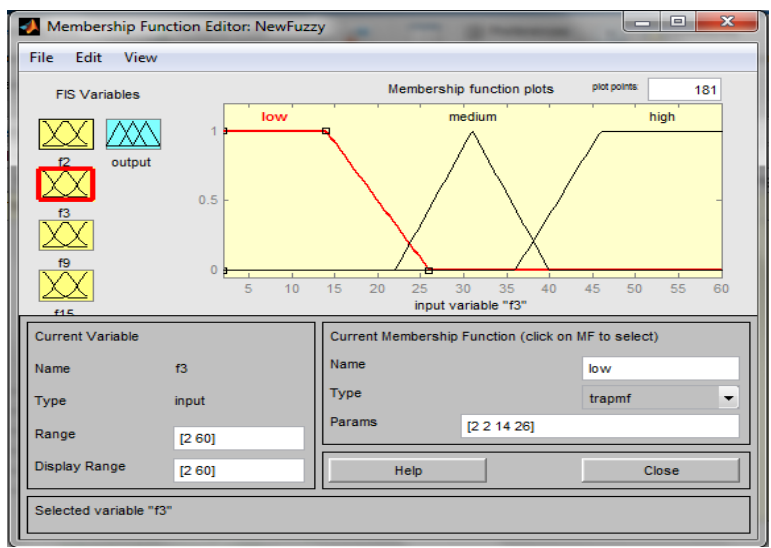

Figure 4 Membership function for feature f3

i.e. ClusterProminence

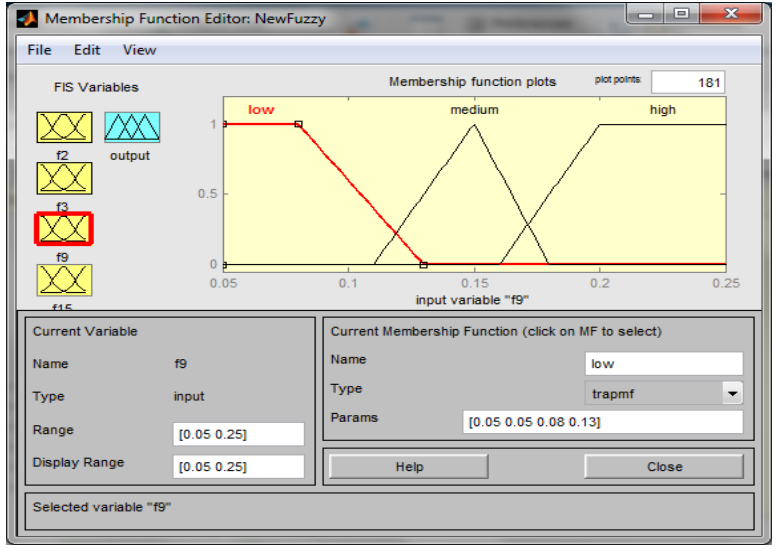

Figure 5 Membership function for feature f9

i.e. Correlation

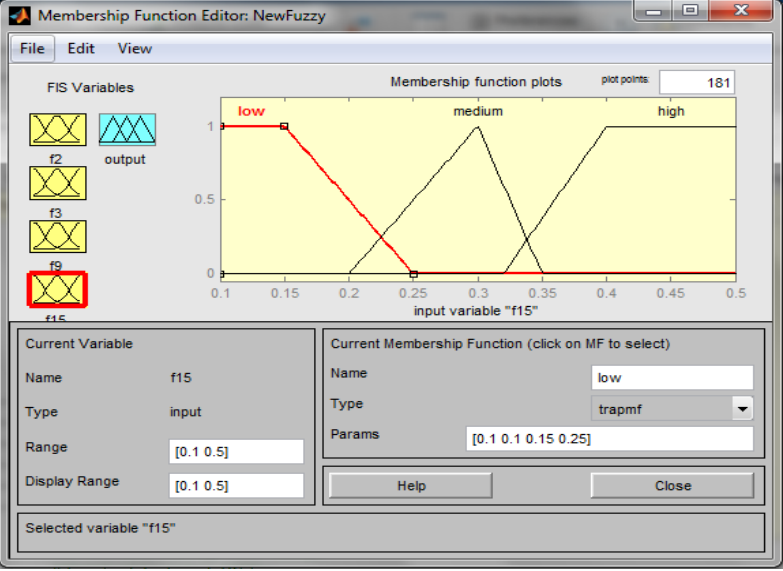

Figure 6 Membership function for feature 15

i.e. differenceEntropy

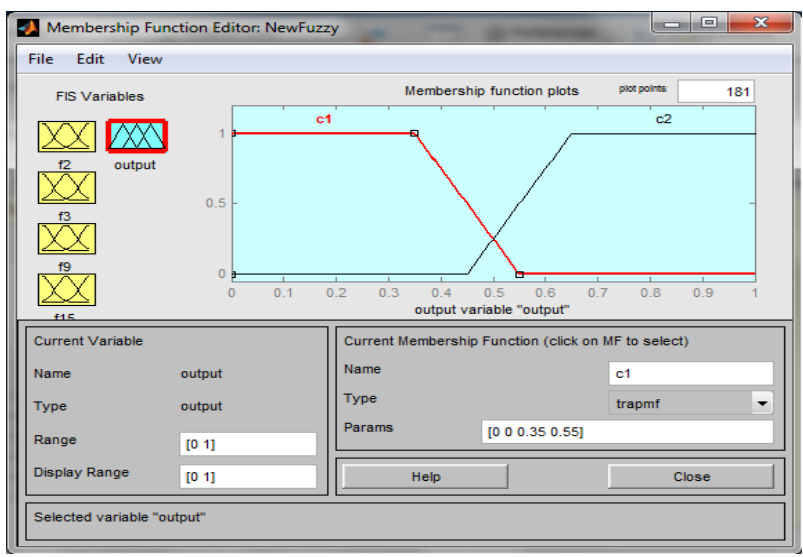

Figure 7 Membership function for output class (benign/malignant)

\subsection{Defuzzification}

The proposed FIUIAS used centroid method for de-fuzzification and is the final step of inferencing. Here a mapping of fuzzy set to crisp set takes place [25-32]. 


\section{RESULTS \& DISCUSSION}

All the above operations are realised by means of Mat lab 14.0. FIUIAS is investigated by means of one output and four input parameters. CBA rules deposited in rule base fires instantaneously for the same input. The structure of designed FIUIAS for diagnosis of TN is displayed in Figure 10. Figures 11 and 12 display rule viewer of FIS to differentiate benign nodule and malignant TNs respectively. The designed FIUIAS method provided classification accuracy of $98 \%$. Results clearly demonstrate fuzzy concepts appear to be more suitable for medical image processing.

\section{CONCLUSION}

To enhance classification accuracy of TN diagnosis, an intelligent FIUIAS system is built using fuzzy concepts. Modelling intelligent system in medical diagnosis field is a difficult task. This is mainly because disease diagnosis requires a proficiency in coping with uncertainty, vagueness and impreciseness. The developed FIUIAS system resulted in an accuracy of $98 \%$ for identification of TNs as benign or malignant. From the outcomes it can be confirmed that, FL can be used as a tool to develop disease diagnosis systems since medical domain is full of vagueness and imprecise conditions. Developed FIUIAS using fuzzy concepts can be operated as supportive means by radiologists in India with vast rural areas and complete scarcity of physicians.

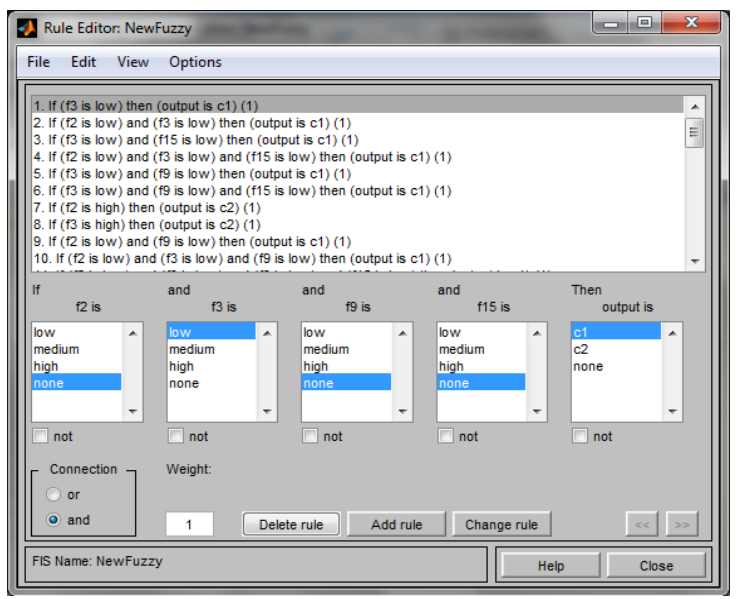

Figure 8 Fuzzy rule base containing CBA rules showing rules firing for class $\mathrm{C} 1$ i.e. benign nodule

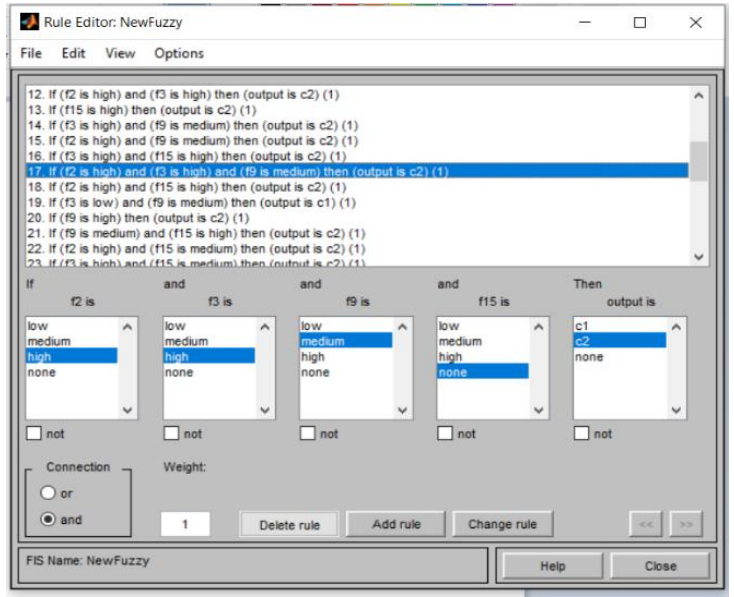

Figure 9 Fuzzy rule base containing CBA rules showing rules firing for class $\mathrm{C} 2$ i.e malignant nodule

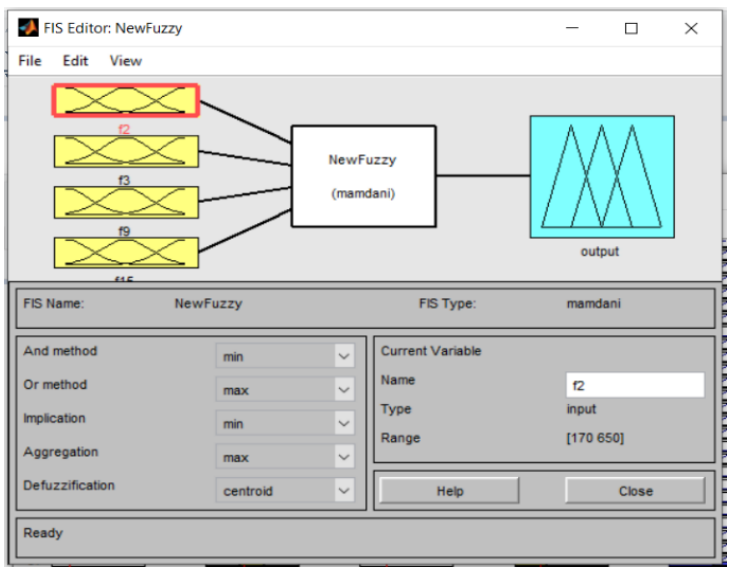

Figure 10 FIUIAS to diagnose TN

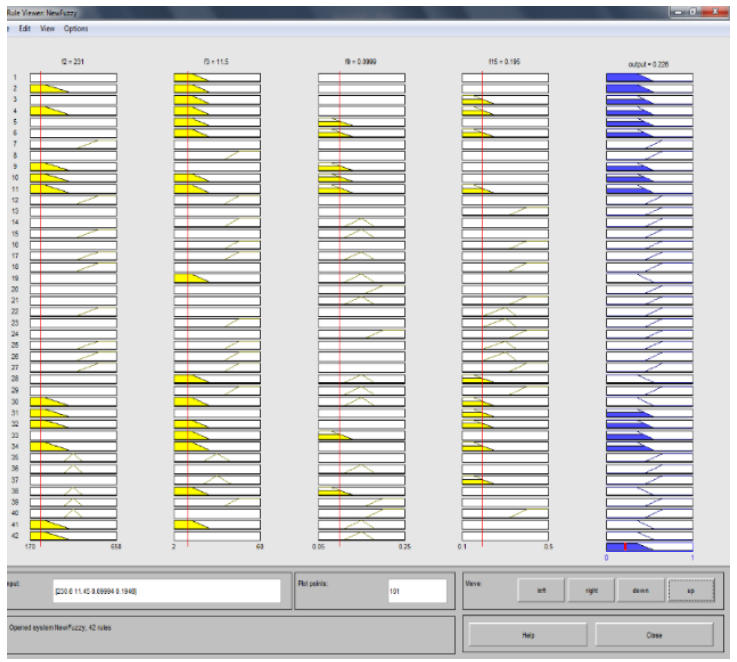

Figure 11 FIUIAS rule viewer for benign TN 


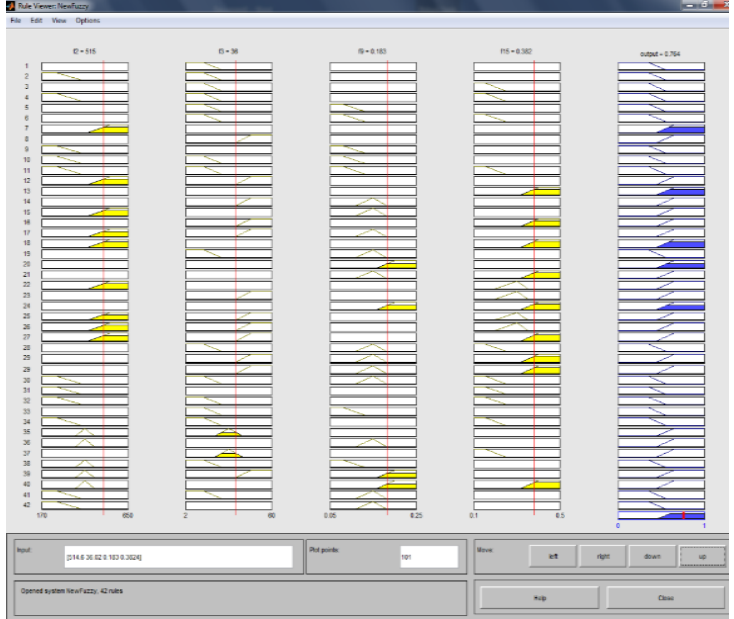

Figure 12 FIUIAS rule viewer for Malignant TN

\section{REFERENCES}

[1] G. Gursel, "Healthcare, uncertainty, and fuzzy logic", Digital Medicine, Vol. 02, No. 03, pp. 101-112, 2016

[2] N. H. Phuong, V. Kreinovich, "Fuzzy Logic and its Applications in Medicine", International Journal of Medical Informatics, Vol. 62, No. 23, pp. 165-173, 2001

[3] J. Greeda, A. Mageswari, R. Nithya, “A Study on Fuzzy Logic and its Applications in Medicine", International Journal of Pure and Applied Mathematics, Vol. 119, No. 16 pp. 1515-1525, 2018

[4] Jimmy Singla, Balwinder Kaur,Deepak Prashar, Sudan Jha, Gyanendra Prasad Joshi, Kyungyun Park, Usman Tariq, Changho Seo, "A Novel Fuzzy Logic-Based Medical Expert System for Diagnosis of Chronic Kidney Disease", Mobile Information Systems, Vol. 2020.

[5] Muralikrishna Puttagunta, S. Ravi, "Medical Image Analysis based on Deep Learning Approach", Multimedia Tools and Applications, Vol 80, pp. 24365-24398, 2021.

[6] Colella Ylenia, De Lauri Chiara, Improta Giovanni, Rossano Lucia, Vecchione Donatella, Spinosa Tiziana, Giordano Vincenzo, Verdoliva Ciro and Santini Stefania, "A Clinical Decision Support System based on fuzzy rules and classification algorithms for monitoring the physiological parameters of type-2 diabetic patients", Mathematical Biosciences and Engineering, Vol. 18, No. 03, pp. 2654-2674, 2021.
[7] Endocrineweb.

http://www.endocrineweb.com/endocrinology/ overview-thyroid

[8] D. E. Maroulis, M. A. Savelonas, D. K. Iakovidis, S. A. Karkanis, N.Dimitropoulos, "Variable Background Active Contour Model for Computer-Aided Delineation of Nodules in Thyroid Ultrasound Images", IEEE Transactions on Information Technology in Biomedicine, Vol. 11, No.05, pp. 537-543, 2007

[9] D. K. Iakovidis, M. A. Savelonas, S. A. Karkanis, D.E. Maroulis, "A Genetically Optimized Level Set Approach to Segmentation of Thyroid Ultrasound Images", Applied Intelligence, Vol. 27, No. 03, pp. 193203, 2007

[10] D. Selvathi, V. S. Sharnitha, "Thyroid Segmentation in Ultrasound Images Using Support Vector Machine", International Journal of Neural Networks and Applications, Vol.04, No. 01, pp. 7-12, 2011

[11] D. Bibicu, L. Moraru, A. Biswas, "Thyroid Nodule Recognition Based on Feature Selection and Pixel Classification Methods", Journal of Digital Imaging, Vol. 26, No. 01, 119-128, 2013

[12] E. G. Keramidas, D. K. Iakovidis, D. Maroulis, S. Karkanis, "Efficient and Effective Ultrasound Image Analysis Scheme for Thyroid Nodule Detection", International Conference on Image Analysis and Recognition, Springer Berlin Heidelberg, 2007

[13] Jianning Chi, Ekta Walia, Paul Babyn, Jimmy Wang, Gary Groot, Mark Eramian, "Thyroid Nodule Classification in Ultrasound Images by Fine-Tuning Deep Convolutional Neural Network", Journal of Digit Imaging, Vol. 30, pp. 477-486, 2017.

[14] E. G. Keramidas, D. K. Iakovidis, D. Maroulis, N. Dimitropoulos, "Thyroid Texture Representation via Noise Resistant Image Features", 21st IEEE International Symposium on Computer-Based Medical Systems, pp. 560565,2008

[15] http://www.mathworks.com

[16] Manfred Blum, "Ultrasonography of the Thyroid", Endotext [Internet], https://www.ncbi.nlm.nih.gov/books/NBK285 $555 /$ 
[17] Cheng Xie, Peter Cox, Nia Taylor, Sarah LaPorte, "Ultrasonography of Thyroid Nodules: A Pictorial Review", Insights Imaging, Vol. 07, No. 01, pp. 77-86, 2016

[18] Digital Database Thyroid Image. http://www.cimalab.unal.edu.co Date accessed $10 / 10 / 2021$

[19] Poornima. D, Asha Gowda Karegowda, P.T.Bharathi, "A Pragmatic Review of Denoising Techniques applied for Medical Images", International Journal of Applied Research on Information Technology and Computing, Vol. 08, No. 01, pp.81-104, 2017

[20] Poornima. D, Asha Gowda Karegowda, "A Review of Image Segmentation Techniques applied to Medical Images", International Journal of Data Mining and Emerging Technologies, Vol. 08, No. 01, pp. 78-94, 2018

[21] T. Lo"fstedt, P. Brynolfsson, T. Asklund, T. Nyholm, A. Garpebring, "Gray-level invariant Haralick texture features", PLOS ONE, Vol. 14, No. 02, 2019

[22] Vipin Kumar, Sonajharia Minz, "Feature Selection: A literature Review", Smart Computing Review, vol. 04, No. 03, pp. 2014

[23] Timothy J. Ross, "Fuzzy Logic with Engineering Applications", Third Edition, John Wiley \& Sons, 2010

[24] Bing Liu, Wynne Hsu, Yiming Ma, "Integrating Classification and Association Rule Mining", Fourth international conference on knowledge discovery and data mining, 1998

[25] V. Kansal, A. Kaur, "Comparison of Mamdani-type and Sugeno type FIS for Water Flow Rate Control in a Rawmill", International Journal of Scientific \& Engineering Research, Vol. 04, No. 06, pp. 2580-2584, 2013

[26] K. Yu, L. Tan, L. Lin, X. Cheng, Z. Yi and T. Sato, "Deep-Learning-Empowered Breast Cancer Auxiliary Diagnosis for 5GB Remote E-Health," IEEE Wireless Communications, vol. 28, no. 3, pp. 54-61, June 2021, doi: 10.1109/MWC.001.2000374.

[27] K. Yu, L. Tan, S. Mumtaz, S. Al-Rubaye, A. Al-Dulaimi, A. K. Bashir, F. A. Khan, "Securing Critical Infrastructures: Deep Learning-based Threat Detection in the IIoT", IEEE Communications Magazine, 2021.

[28] K. Yu, Z. Guo, Y. Shen, W. Wang, J. C. Lin, T. Sato, "Secure Artificial Intelligence of Things for Implicit Group Recommendations", IEEE Internet of Things Journal, 2021, doi: 10.1109/JIOT.2021.3079574.

[29] H. Li, K. Yu, B. Liu, C. Feng, Z. Qin and G. Srivastava, "An Efficient Ciphertext-Policy Weighted Attribute-Based Encryption for the Internet of Health Things," IEEE Journal of Biomedical and Health Informatics, 2021, doi: 10.1109/JBHI.2021.3075995.

[30] L. Tan, K. Yu, F. Ming, X. Cheng, G. Srivastava, "Secure and Resilient Artificial Intelligence of Things: a HoneyNet Approach for Threat Detection and Situational Awareness", IEEE Consumer Electronics Magazine, 2021, doi: 10.1109/MCE.2021.3081874.

[31] L. Tan, N. Shi, K. Yu, M. Aloqaily, Y. Jararweh, "A Blockchain-Empowered Access Control Framework for Smart Devices in Green Internet of Things", ACM Transactions on Internet Technology, vol. 21, no. 3, pp. 1-20, 2021,https://doi.org/10.1145/3433542.

[32] Puttamadappa, C., and B. D. Parameshachari. "Demand side management of small scale loads in a smart grid using glow-worm swarm optimization technique." Microprocessors and Microsystems 71 (2019): 102886.

[33] Subramani, Prabu, Ganesh Babu Rajendran, Jewel Sengupta, Rocío Pérez de Prado, and Parameshachari Bidare Divakarachari. "A block bi-diagonalization-based pre-coding for indoor multiple-input-multiple-output-visible light communication system." Energies 13, no. 13 (2020): 3466

[34] Kumar, M. Keerthi, B. D. Parameshachari, S. Prabu, and Silvia liberata Ullo. "Comparative Analysis to Identify Efficient Technique for Interfacing BCI System." In IOP Conference Series: Materials Science and Engineering, vol. 925, no. 1, p. 012062. IOP Publishing, 2020.

[35] Hu, Liwen, Ngoc-Tu Nguyen, Wenjin Tao, Ming C. Leu, Xiaoqing Frank Liu, Md Rakib Shahriar, and SM Nahian Al Sunny. "Modeling of cloud-based digital twins for smart manufacturing with MT connect." Procedia manufacturing 26 (2018): 1193-1203.

[36] Seyhan, Kübra, Tu N. Nguyen, Sedat Akleylek, Korhan Cengiz, and SK Hafizul Islam. "BiGISIS KE: Modified key exchange protocol with reusable keys for IoT security." Journal of Information Security and Applications 58 (2021): 102788. 
[37] Nguyen, Tu N., Bing-Hong Liu, Nam P. Nguyen, and Jung-Te Chou. "Cyber security of smart grid: attacks and defenses." In ICC 20202020 IEEE International Conference on Communications (ICC), pp. 1-6. IEEE, 2020.

[38] Arun, M., E. Baraneetharan, A. Kanchana, and S. Prabu. "Detection and monitoring of the asymptotic COVID-19 patients using IoT devices and sensors." International Journal of Pervasive Computing and Communications (2020).

[39] Kumar, M. Keerthi, B. D. Parameshachari, S. Prabu, and Silvia liberata Ullo. "Comparative Analysis to Identify Efficient Technique for Interfacing BCI System." In IOP Conference Series: Materials Science and Engineering, vol. 925, no. 1, p. 012062. IOP Publishing, 2020. 